Supporting Information

\title{
Rapid oxidation following photoreduction in the avian cryptochrome4 photocycle
}

Hiroaki Otsuka ${ }^{1}$,Hiromasa Mitsui ${ }^{1}$, Kota Miura ${ }^{1}$, Keiko Okano $^{1}$, Yasushi Imamoto $^{2}$, and Toshiyuki Okano ${ }^{1 *}$.

${ }^{1}$ Department of Electrical Engineering and Bioscience, Graduate School of Sciences and Engineering, Waseda University,

TWIns, Wakamatsucho 2-2, Shinjuku-Ku, Tokyo 162-8480, Japan;

${ }^{2}$ Department of Biophysics, Division of Biological Sciences, Graduate School of Science, Kyoto University, Kitashirakawa-Oiwakecho, Sakyo-Ku, Kyoto 606-8502, Japan. 


\section{Supplementary Figure S1}
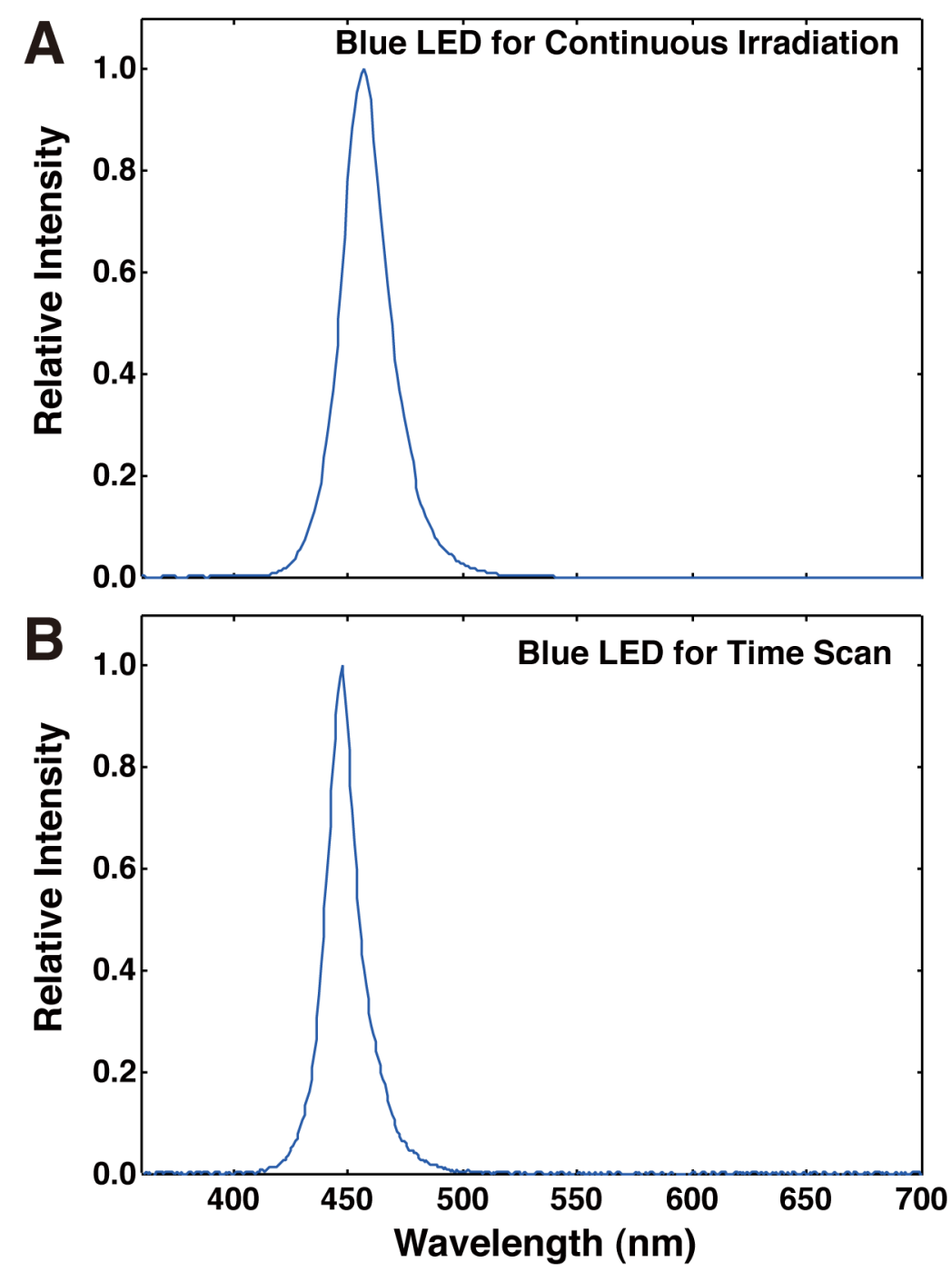

Figure S1. Relative intensity of LEDs for the excitation of cCRY4. (A) Relative intensity of the blue LED light used for continuous irradiation of cCRY4 (Figure 1). The blue LED of a three colors LED (OptoSupply, OSTCXBCBC1E, $\lambda_{\max }=457 \mathrm{~nm}, 1.2 \mathrm{~mW} / \mathrm{cm}^{2}$ ) was used. (B) Relative intensity spectrum of the blue LED (LXK2-PR14-R00, $\lambda_{\max }=448 \mathrm{~nm}, 4.8 \mathrm{~mW} / \mathrm{cm}^{2}$ ) used in the time scan measurement (Supplementary Figure S7). Spectra were measured using a photonic multichannel spectral analyzer (Hamamatsu Photonics, Model PMA-11, type C7473-36). 


\section{Supplementary Figure S2}
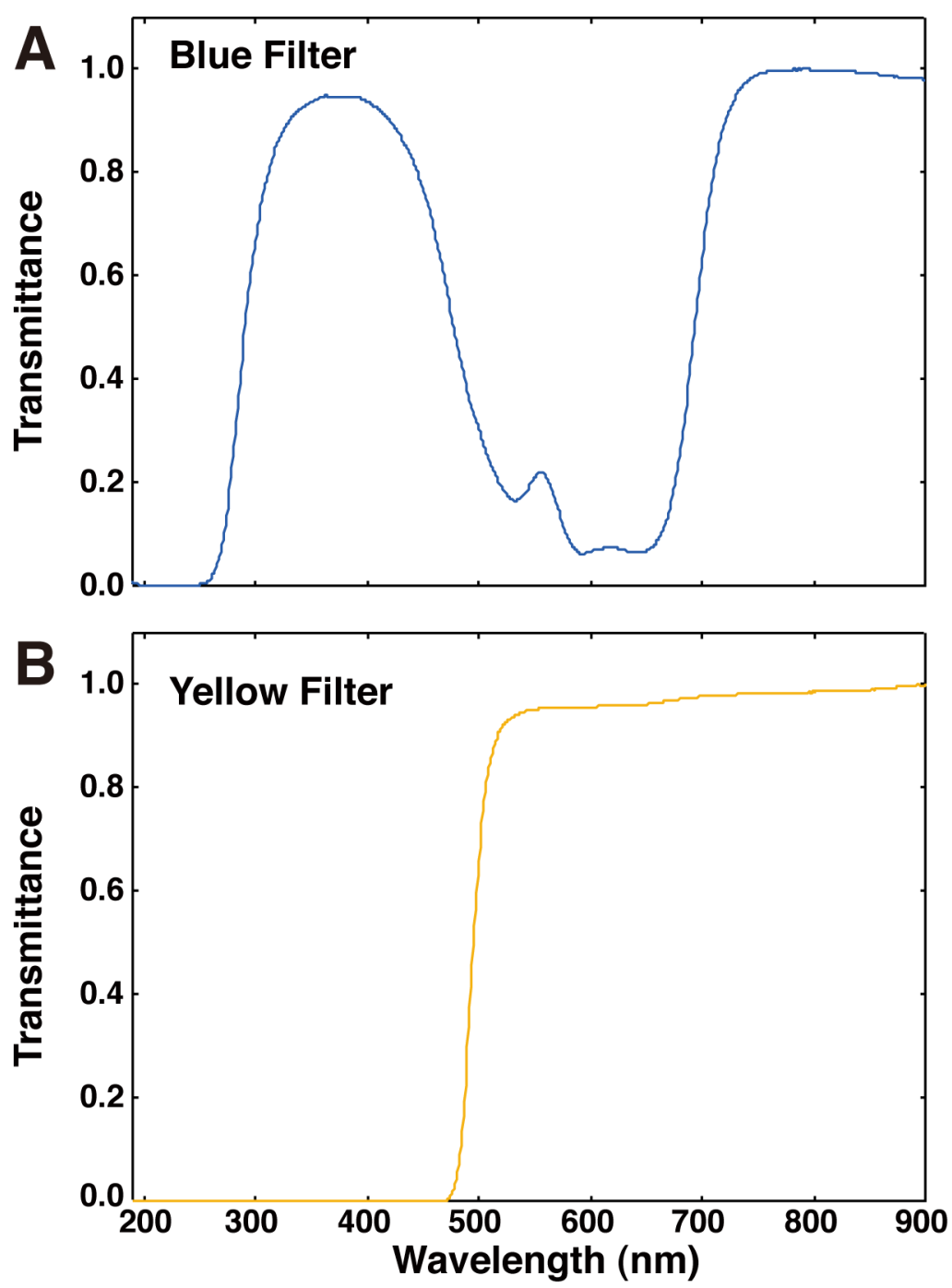

Figure S2. The transmission spectra of filters and the relative intensity of the red LED used in flash photolysis. (A) Transmission spectrum of the blue filter used for the photoexcitation of FAD $_{\mathrm{OX}}$. (B) Transmission spectrum of the yellow filter (Y49) used for photoexcitation of $\mathrm{FADH}^{\bullet}$. Spectra were measured using a UV-2450 spectrophotometer (Shimadzu). 


\section{Supplementary Figure S3}
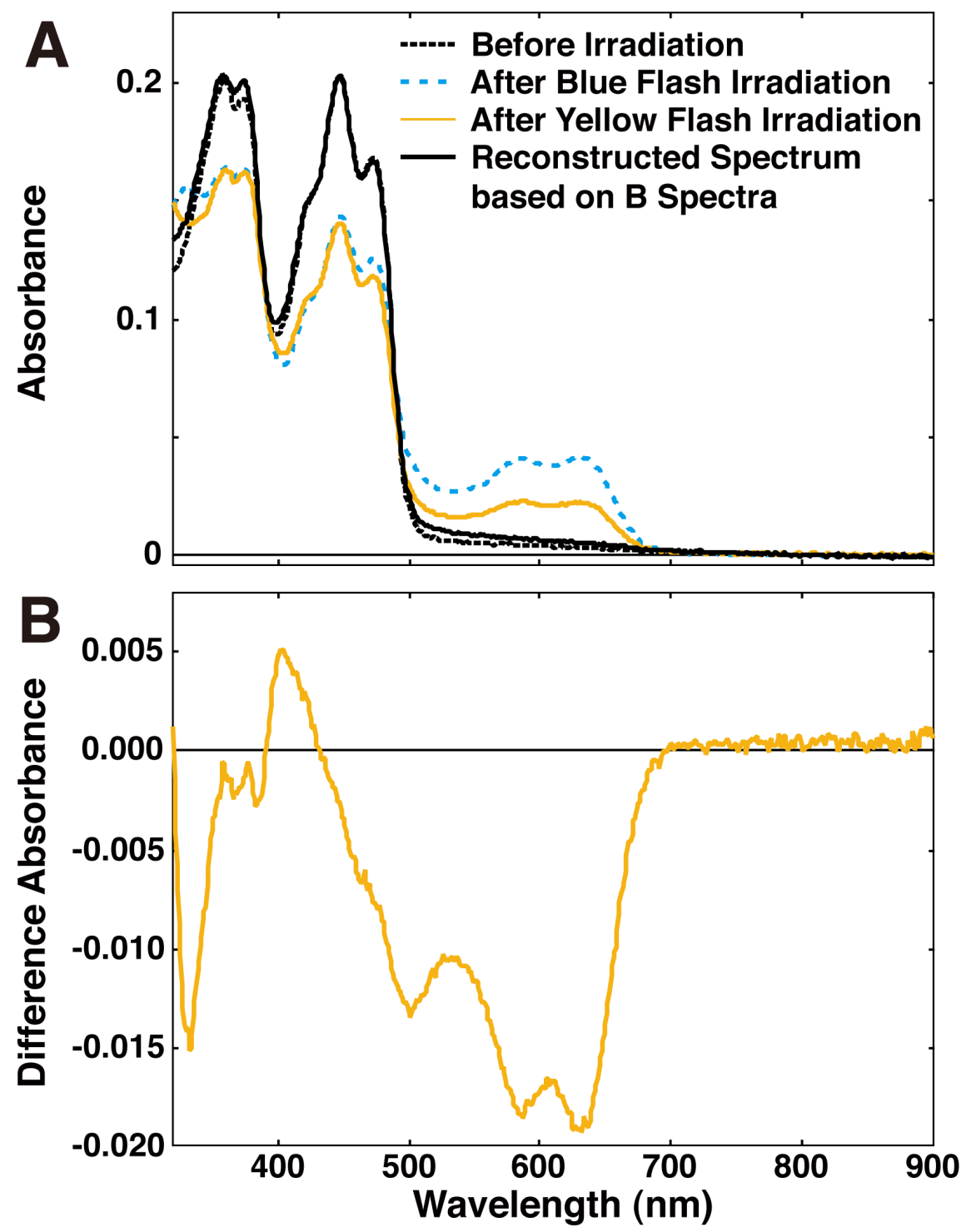

Figure S3. UV-Vis spectra after flash photolysis. (A) Black broken curve and blue broken curve denote UV-Vis spectra of the cCRY4 sample used in flash photolysis (Figure 3), under dark and after blue flash, respectively. Orange curve denotes the spectrum after the photoreaction of $\mathrm{FADH}^{\bullet}$ to $\mathrm{FADH}^{-}$, as driven by the yellow flash. (B) Difference spectrum between the spectra before and after the yellow flash (orange curve in panel A - blue broken curve in panel A). 


\section{Supplementary Figure S4}

A

\section{INVSc1 yeast cells expressing GST-PS-cCRY4}

$\downarrow$ Breaking the cells by sonication

Crude extract

$\downarrow$ Loading on Glutathione-Sepharose column

Washing the column with GST column buffer,

and eluting with GST elution buffer

\section{GST-PS-cCRY4}

$\downarrow$ Dialysis against GST column buffer

$\downarrow$ Cleavage of GST tag with PreScission protease

\section{Mixture of cCRY4, GST tag, and PreScission protease}

Loading on Glutathione-Sepharose column to trap GST tag

$\downarrow$ and PreScission protease (having GST tag)

$\downarrow$ Collect the flow-through fraction

\section{Untagged cCRY4}

B

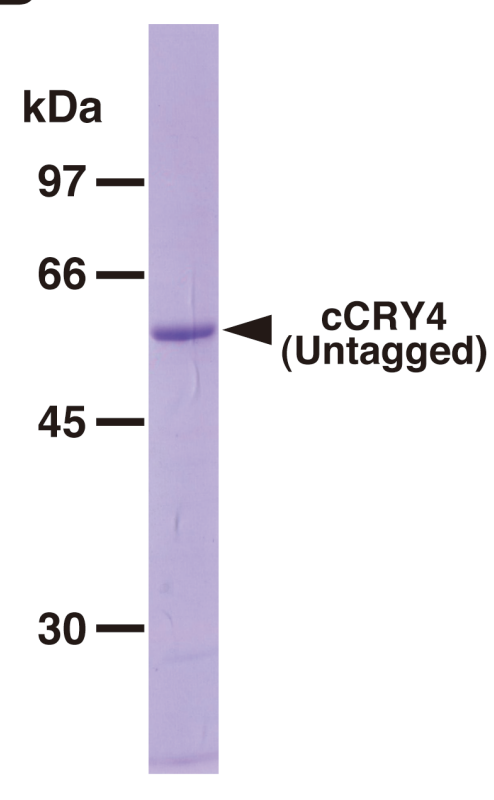

Figure S4. The purification of cCRY4. (A) The scheme of the purification of cCRY4. After the purification of GST-PS-cCRY4 by Glutathione Sepharose 4B resin, the GST tag was cleaved by PreScission protease. (B) Coomassie blue staining of cCRY4. The arrow indicates the band of cCRY4 (61 kDa). 


\section{Supplementary Figure S5}
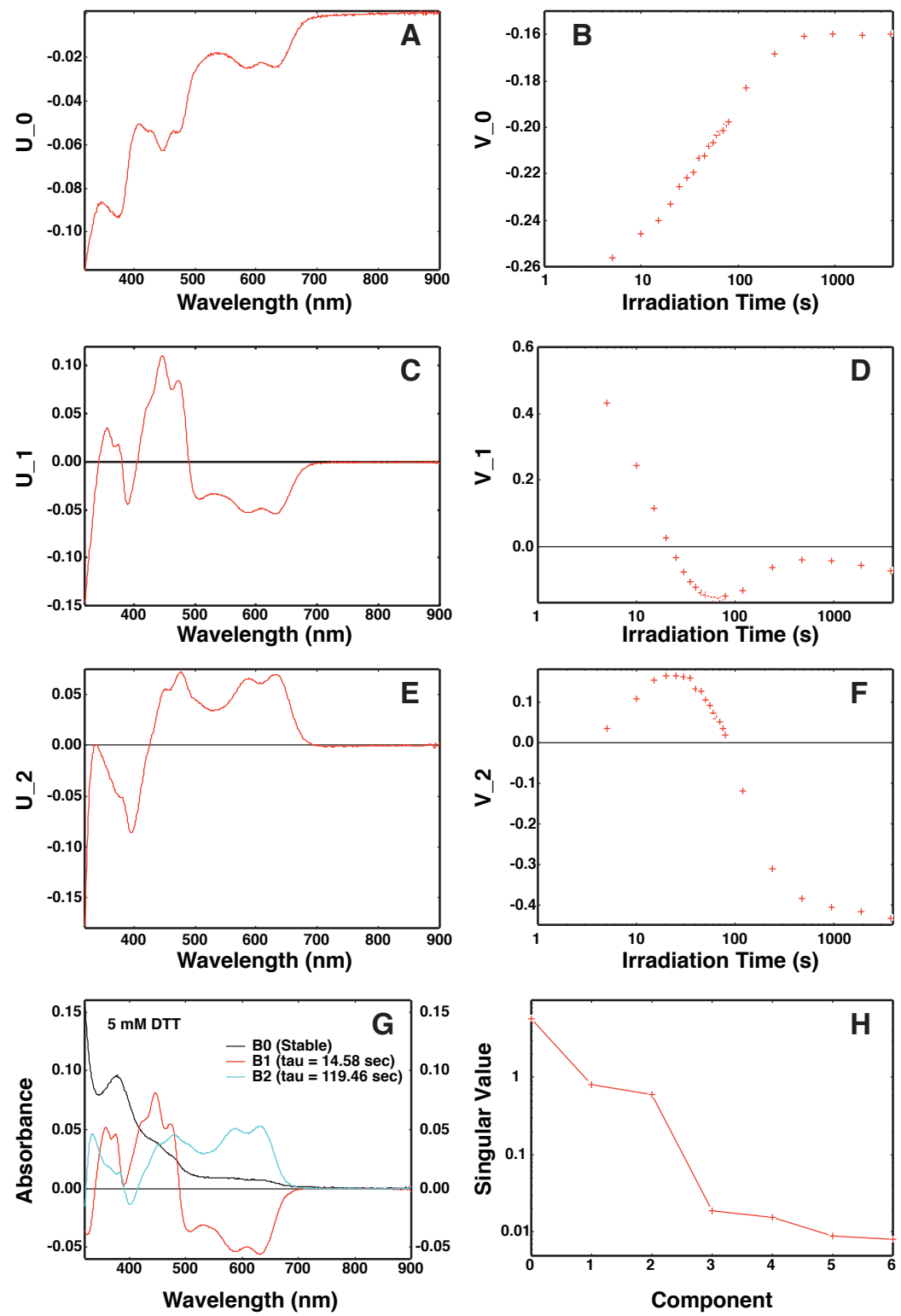

Figure S5. SVD analysis of the UV-Vis spectroscopic data of cCRY4 irradiated with blue light in the presence of $5 \mathrm{mM}$ DTT. SVD analysis of the photoreaction of cCRY4 in the presence of 5 mM DTT (Figure 1A). (A, C, E) Calculated U spectra. (B, D, F) Plots of calculated V spectra. (G) Reconstructed B spectra. (H) Singular value plot for each U and V spectral pair. 


\section{Supplementary Figure S6}

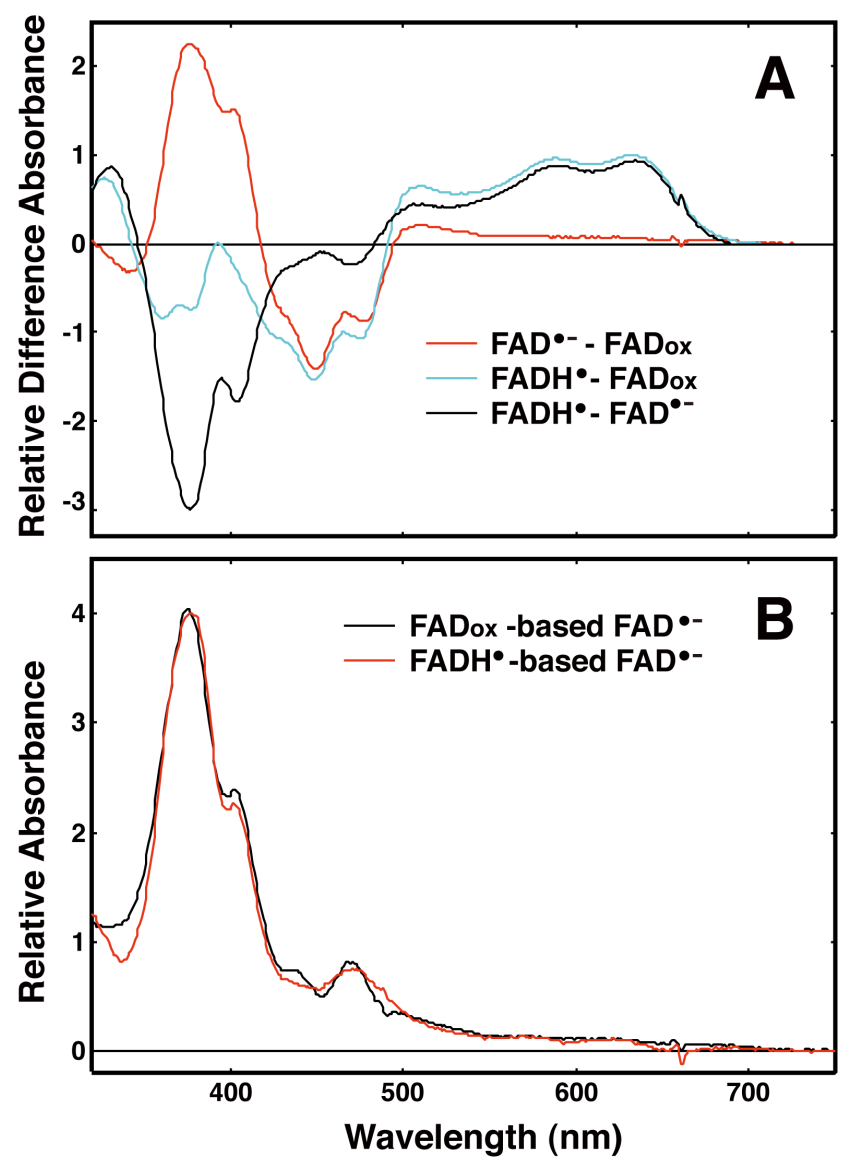

Figure S6. Calculation of the absolute absorbance of $\mathrm{FAD}^{\bullet-}$. (A) Red curve, a difference spectrum obtained $0.5 \mathrm{~ms}$ after flashed light, in the presence of DTT (reproduced from Fig. 4A). (B) FAD Ox $^{\text {-based }} \mathrm{FAD}^{\bullet-}$ spectrum $\left(\alpha=0.976\right.$, red curve) and FADH॰-based $\mathrm{FAD}^{\bullet-}$ spectrum $(\beta=0.974$, black curve $)$ determined to give the minimum sum of squares of absorption differences (for detail, see Supplementary Table S1). 


\section{Supplementary Figure S7}

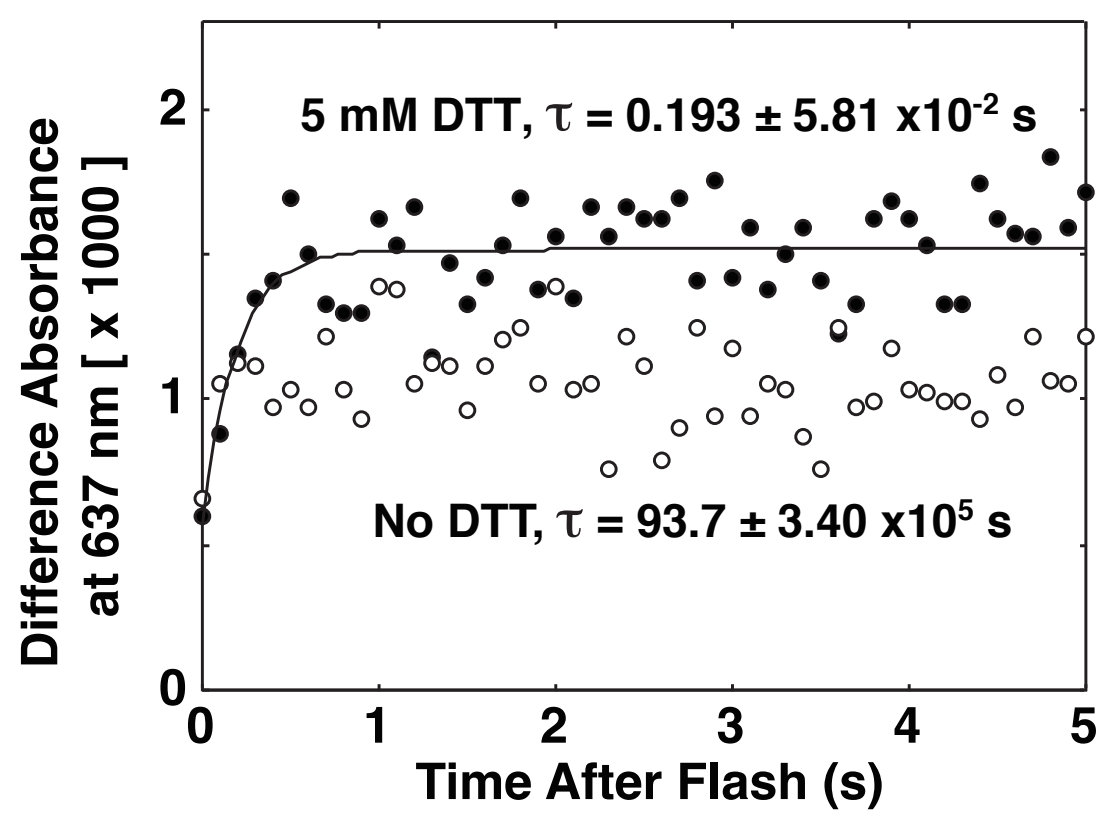

Figure S7. Time scan measurement of cCRY4 photoreduction. Absorbance of the sample was recorded every $0.1 \mathrm{~s}$ after blue LED flash (Supplementary Figure S1B), in the presence (filled circles) or absence (open circles) of DTT, at $20^{\circ} \mathrm{C}$. Exponential fitting was performed using Igor Pro 6.22J. 


\section{Supplementary Figure S8}
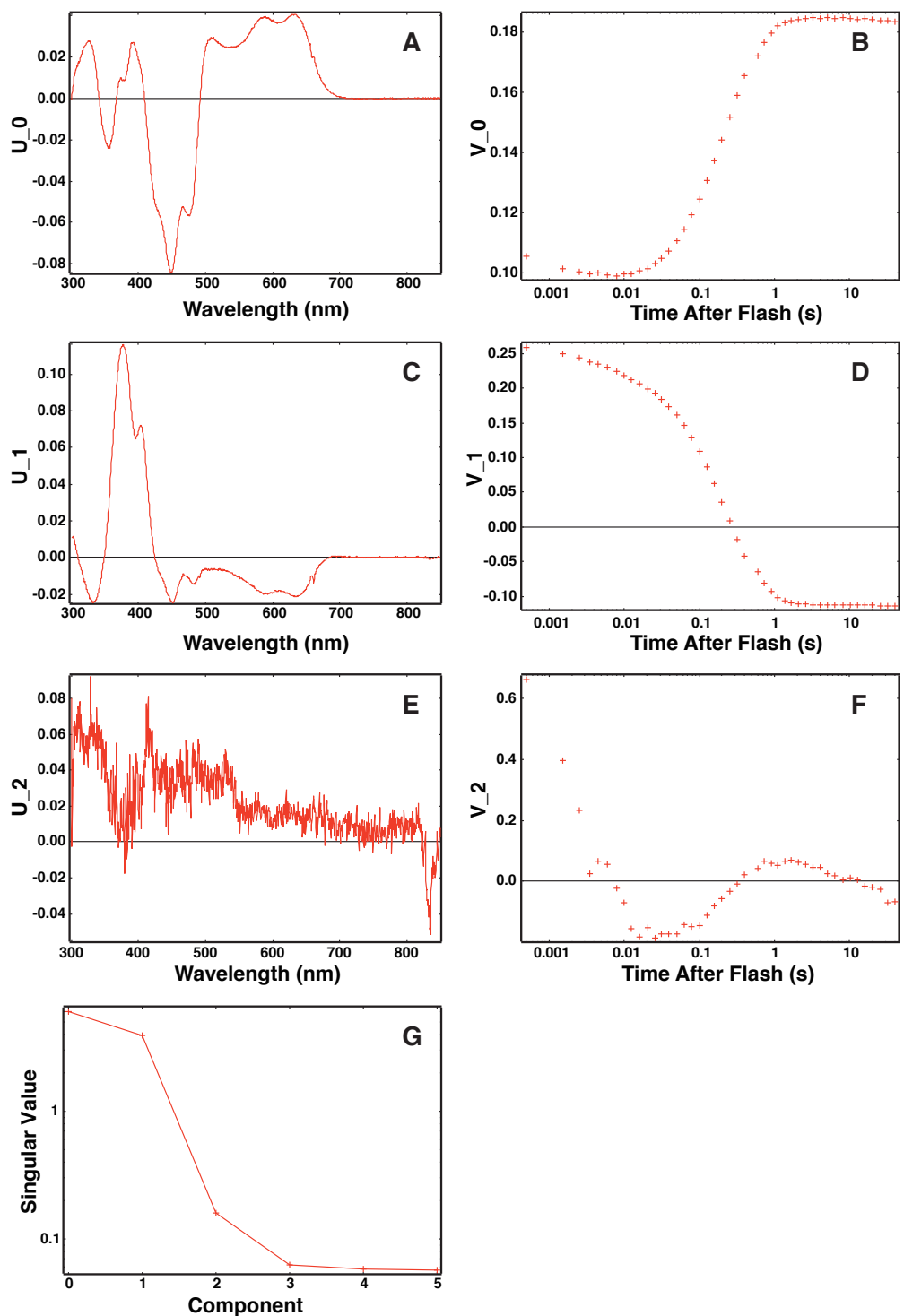

Figure S8. SVD analysis of millisecond flash spectroscopic data of the cCRY4 FAD ${ }_{\mathrm{Ox}}$ form, irradiated with blue flash, in the presence of $5 \mathrm{mM}$ DTT. SVD analysis of the transient photoreaction of cCRY4 in the presence of $5 \mathrm{mM}$ DTT (Figure 3A). (A, C, E) Calculated U spectra. (B, D, F) Plots of calculated V spectra. (G) Singular value plot for each U and V spectral pair. 


\section{Supplementary Figure S9}
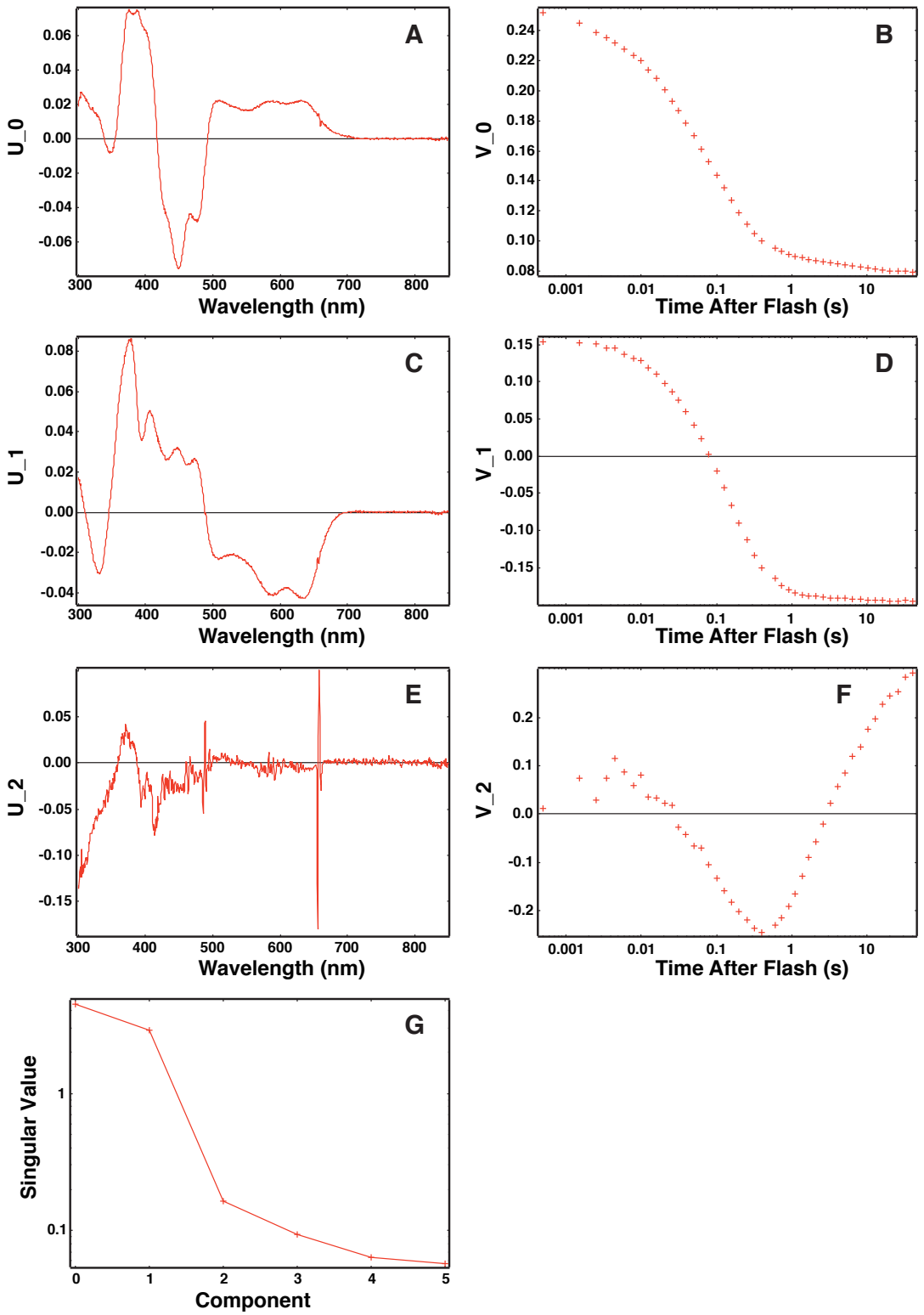

Figure S9. SVD analysis of the millisecond flash spectroscopic data of cCRY4, irradiated with blue flash, in the absence of $5 \mathrm{mM}$ DTT. SVD analysis of the transient photoreaction of cCRY4 in the absence of $5 \mathrm{mM}$ DTT (Figure 3B). (A, C, E) Calculated U spectra. (B, D, F) Plots of calculated V spectra. (G) Singular value plot for each U and V spectral pair. 


\section{Supplementary Figure S10}
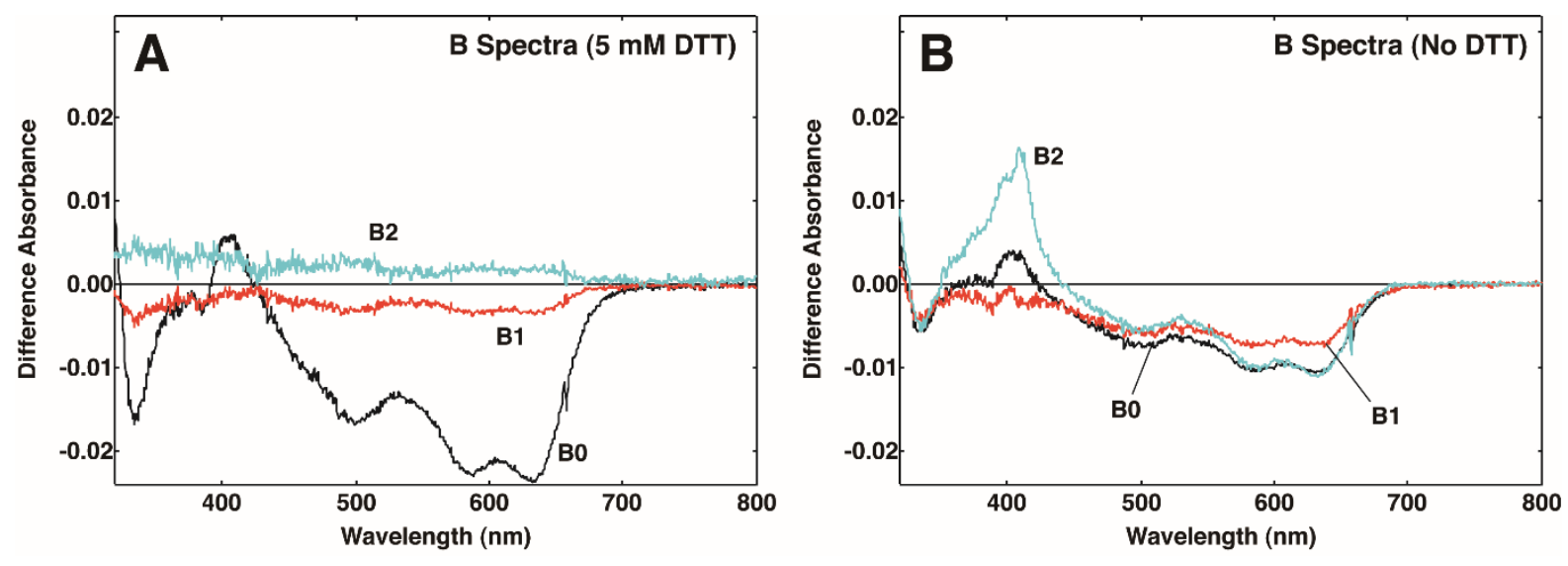

Figure S10. B spectra in SVD analysis of data from the photoreaction $\mathrm{FADH}^{\bullet}$ to $\mathrm{FADH}^{-}$in the presence or absence of DTT (Figure 4A and 4B). (A) B spectra in the presence of DTT. The B0 spectrum is the steady-state spectrum (black curve). The time constants of B1 (red curve) and B2 (blue curve) spectra were calculated to be $275 \mathrm{~ms}$ and $2.92 \mathrm{~s}$, respectively. (B) B spectra in the absence of DTT. The B0 spectrum is the steady-state spectrum (black curve). The time constants of B1 (red curve) and B2 (blue curve) spectra were calculated to be $114 \mathrm{~ms}$ and $1.85 \mathrm{~s}$, respectively. Data in time regions from $0.5 \mathrm{~ms}$ to $50 \mathrm{~s}$, after flash irradiation, were analyzed. 


\section{Supplementary Table S1}

Table S1. Procedures performed in the calculation of absolute absorption of FAD ${ }^{\bullet-}$.

\begin{tabular}{|c|c|}
\hline & Procedures \\
\hline 1 & $\begin{array}{l}\text { A difference spectrum, corresponding to } 0.5 \mathrm{~ms} \text { after flashed light in the presence of DTT, } \\
\text { was calculated as the sum of B0, B1, and B2 (Figure } 3 \mathrm{C} \text { ), and considered to be a difference } \\
\text { spectrum between } \mathrm{FAD}^{\bullet-} \text { and } \mathrm{FAD}_{\mathrm{Ox}}\left(\text { termed }\left[\mathrm{FAD}^{\bullet-}-\mathrm{FAD}_{\mathrm{Ox}}\right] \text { ). }\right.\end{array}$ \\
\hline 2 & $\begin{array}{l}\text { The B0 spectrum, calculated in the flash photolysis (black curve; Figure } 3 \mathrm{C} \text { ), was the steady } \\
\text { state spectrum, and was considered the difference spectrum between FADH }{ }^{\bullet} \text { and FADox. } \\
\text { (termed }\left[\mathrm{FADH}^{\bullet}-\mathrm{FAD}_{\mathrm{OX}}\right] \text { ). }\end{array}$ \\
\hline 3 & $\begin{array}{l}{\left[\mathrm{FAD}^{\bullet-}-\mathrm{FAD}_{\mathrm{Ox}}\right] \text { and }\left[\mathrm{FADH}^{\bullet}-\mathrm{FAD}_{\mathrm{Ox}}\right] \text { were divided using the absorbance at } 632 \mathrm{~nm} \text { of }} \\
{\left[\mathrm{FADH}^{\bullet}-\mathrm{FAD}_{\mathrm{Ox}}\right] \text {, so that the absorbance of } \mathrm{FADH}^{\bullet} \text { at } 632 \mathrm{~nm} \text { was } 1 \text { (rescaling for }} \\
\text { normalization) (Supplementary Figure S6A, red curve, and blue curve, respectively). }\end{array}$ \\
\hline 4 & $\begin{array}{l}\quad\left[\mathrm{FAD}^{\bullet-}-\mathrm{FAD}_{\mathrm{Ox}}\right] \text { was subtracted from }\left[\mathrm{FADH}^{\bullet}-\mathrm{FAD}_{\mathrm{Ox}}\right] \text { to calculate the difference } \\
\text { spectrum between } \mathrm{FADH}^{\bullet} \text { and } \mathrm{FAD}^{\bullet-}\left(\text { termed }^{\circ} \mathrm{FADH}^{\bullet}-\mathrm{FAD}^{\bullet}\right] \text {, Supplementary Figure S6A, } \\
\text { black curve). }\end{array}$ \\
\hline 5 & $\begin{array}{l}{\left[\mathrm{FAD}^{\bullet-}-\mathrm{FAD}_{\mathrm{Ox}}\right] \text { and }\left[\mathrm{FADH}^{\bullet}-\mathrm{FAD}^{\bullet-}\right] \text { were rescaled for normalization, so that absorbance }} \\
\text { of }\left[\mathrm{FADH}^{\bullet}-\mathrm{FAD}^{\bullet-}\right] \text { at } 632 \mathrm{~nm} \text { was } 1 .\end{array}$ \\
\hline 6 & $\begin{array}{l}\text { The absolute absorption spectra for } \mathrm{FADH}^{\bullet} \text { and } \mathrm{FAD}_{\mathrm{Ox}} \text { (Figure } 2 \text {, red curve and black curve, } \\
\text { respectively) were multiplied by } 1.93 \text {, so that the absorbance of } \mathrm{FADH}^{\bullet} \text { at } 632 \mathrm{~nm} \text { was } 1 \\
\text { (rescaling for normalization). }\end{array}$ \\
\hline 7 & $\begin{array}{l}\text { The normalized }\left[\mathrm{FADH}^{\bullet}-\mathrm{FAD}^{\bullet-} \text { ] was multiplied by variable } \alpha(0<\alpha<1) \text { and subtracted }\right. \\
\text { from the normalized absolute absorption spectrum of } \mathrm{FADH}^{\bullet} \text {, because the true ratio between } \\
\text { the molar extinction coefficients of FADH }{ }^{\bullet} \text { and FAD } \mathrm{FAD}^{\bullet-} \text { was unknown. Here, variable } \alpha \text { was } \\
\text { increased from } 0 \text { to } 1 \text { by } 0.002 \text { to obtain the putative absolute absorption spectra of } \mathrm{FAD}^{\bullet-} \\
\text { (termed FADH }{ }^{\bullet} \text {-based } \mathrm{FAD}^{\bullet-} \text { spectra). }\end{array}$ \\
\hline 8 & 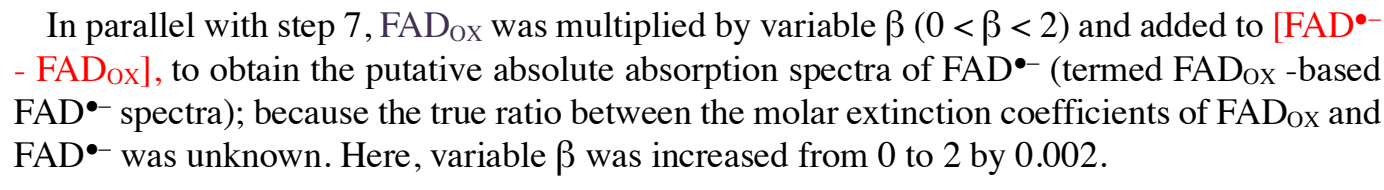 \\
\hline 9 & $\begin{array}{l}\text { An optimal pair of } \alpha \text { and } \beta \text {, that gives the minimal difference degree (see below) between } \\
\text { FADH }^{\bullet-\text { based FAD }}{ }^{\bullet-} \text { spectra and FAD } \text { ox }^{- \text {based FAD }}{ }^{\bullet-} \text { spectra, was determined by calculating } \\
\text { all possible combinations of } \alpha \text { and } \beta \text { for } 500 \text { FADH }^{\bullet} \text {-based FAD }{ }^{\bullet-} \text { spectra and } 1000 \text { FAD }^{-} \text {Ox }^{-} \\
\text {based FAD }{ }^{\bullet-} \text { spectra, respectively. The difference degree was calculated by summing the } \\
\text { squares of absorption differences between the two spectra at wavelengths in a range from } 320 \\
\text { nm to } 900 \mathrm{~nm} \text {, every } 1 \mathrm{~nm} \text {. Next, the optimal pair }[\alpha, \beta] \text { was determined to be [0.976, } 0.974] \text {. }\end{array}$ \\
\hline 10 & 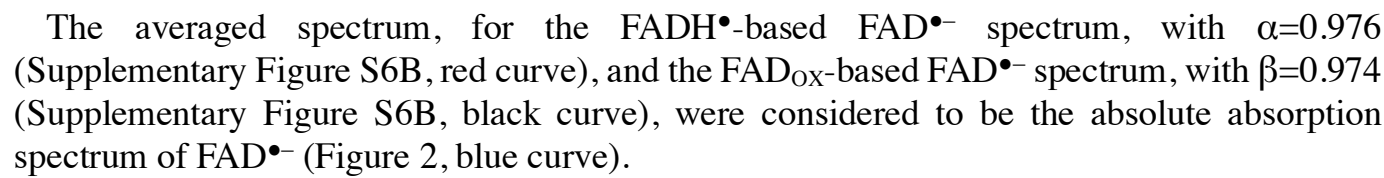 \\
\hline
\end{tabular}

